\title{
Internacionalización, Tramas Productivas y Sistema Nacional de Innovación
}

\author{
Hernán Alejandro Morero'
}

\begin{abstract}
This paper aims to study the capacity of the National Systems of Innovation to affect the innovative performance of firms in internationalized production activities of a developing economy. The research adopted a production networks perspective on Innovation Systems and the empirical work involved a survey to firms from the automotive productive network in Argentina. The importance of domestic and external sources of knowledge to the innovative performance of these firms was evaluated through the use of multivariate analysis and data clustering techniques. The main findings of the study are that: i) the innovative performance of argentinian auto parts firms is positively related to certain complementation between internal and external sources of knowledge; and that ii) the importance of the national innovation system is essential for them, even if those firms belongs to internationalized production networks that operates in a developing country.
\end{abstract}

Key words: internationalization; national system of innovation; production networks; automotive production network; Argentina.

\section{Resumen}

El presente artículo tiene el objetivo de estudiar la capacidad de los Sistemas Nacionales de Innovación de afectar el desempeño innovador de las empresas en actividades productivas internacionalizadas de una economía en desarrollo. Para ello se adoptó una perspectiva de Sistemas de Innovación de tramas productivas y se realizó un relevamiento a empresas de la trama automotriz en Argentina. A través del uso de técnicas de análisis multivariante de datos y de técnicas de cluster, se evaluó la importancia de las fuentes domésticas y externas de conocimiento en el desempeño innovador de estas empresas. Las principales conclusiones del estudio son que: i) el desempeño innovador de las empresas autopartistas argentinas está positivamente relacionado con determinada complementación entre fuentes internas y externas de conocimiento; y ii) la importancia del Sistema Nacional de Innovación es esencial para ellas, incluso siendo parte de tramas internacionalizadas que operan en un país en desarrollo.

Palabras Clave: internacionalización; sistema nacional de innovación; tramas productivas; trama productiva automotriz; Argentina.

\footnotetext{
I Centro de Estudios Avanzados - CONICET, Universidad Nacional de Córdoba (Gral. Paz 154, $2^{\circ}$ Piso, Córdoba, Argentina, CP 5000; Tel.: +54 35I 434 I I 24) y Departamento de Economía y Finanzas, Facultad de Ciencias Económicas, Universidad Nacional de Córdoba (Valparaíso S/N, Ciudad Universitaria, Córdoba, Argentina, CP: 5000; Tel. +54 35I 443 7300). E-mail: hernanmorero@eco.uncor.edu
} 


\section{Introducción}

El desempeño económico de una nación está fuertemente afectado por la tasa y la dirección de la innovación. Éstas dependen de la co-evolución entre los distintos aspectos de la configuración institucional y de la estructura económica de la nación, las dimensiones que hacen al Sistema Nacional de Innovación (SNI). En las últimas décadas las características salientes del contexto económico y tecno-productivo involucran un grado creciente de internacionalización: una gran expansión de los flujos internacionales de comercio, capitales, tecnología e información, una mayor interconexión entre los sistemas productivos y socio-institucionales de las naciones, y una importante transnacionalización de los actores económicos. Una preocupación recurrente en la literatura de SNI es la manera en que el carácter de nacional de los Sistemas de Innovación (SI) se ve afectado por este fenómeno de internacionalización de la producción $y$, dentro de esta problemática, la relevancia que en este contexto adquieren las fuentes domésticas de obtención de conocimiento para la innovación (Lundvall, 2007, 1992; Johnson, 1992; Nelson, 1993; López, 1996; Chudnosky, 1999; Balzat y Hanusch 2004).

La bibliografía sobre internacionalización y Sls, puede agruparse en cuatro conjuntos de estudios ${ }^{2}$ : i) un conjunto de estudios sobre las barreras a la internacionalización de los SI, particularmente la concentración espacial de algunas actividades tecnológicas (Mowery y Oxlet, 1997; Mytelka, 2000; Sung y Carlsson, 2003); ii) otro grupo de estudios sobre el grado de internacionalización de las actividades corporativas de I+D como parte del SNI, que encuentran que el grado de internacionalización de estas actividades ha crecido en las últimas décadas, particularmente desde los años noventa conducida por el desarrollo de redes corporativas (Cantwell, 1997; Cantwell y Piscitello, 2000; Carlsson y Mudambi, 2003; Le bas y Sierra, 2002; Patel y Pavitt, 199I, 1999); iii) un grupo de estudios empíricos que examinan el grado de internacionalización de los SNI, concentrados en casos de países desarrollados (Niosi y Bellon, 1994, 1996; Bartholomew, 1997; Fransman, 1999); y iv) un grupo de estudios sobre la importancia de los SNI en este contexto de internacionalización que, basándose también en países desarrollados, coinciden en afirmar que pese a la internacionalización, los SNI siguen siendo determinantes esenciales en el desempeño innovador de las empresas (Patel, 1997; Freeman, 1995; Pavitt, 1998; Foray, 1995).

En este contexto, en el presente artículo se indaga si en actividades productivas internacionalizadas de una economía en desarrollo, que se encuentra alejada de la frontera económica internacional, las fuentes nacionales de obtención de conocimiento son relevantes para explicar la performance innovativa de las firmas o si, por el contrario, su importancia es marginal. Para ello, se ha diseñado un enfoque de SNI desde una perspectiva de tramas productivas, $y$ se ha seleccionado una trama productiva internacionalizada (la trama automotriz), en un país en desarrollo (la Argentina), para evaluar la importancia relativa de las fuentes domésticas y externas de conocimiento en el desempeño innovador de las empresas, a través del uso de técnicas de análisis multivariante de datos.

El artículo se estructura del siguiente modo: en la próxima sección presentamos el marco teórico (el marco de SNI, las distintas perspectivas $y$ el enfoque de tramas) $y$ precisamos el planteo del problema y objetivos; luego en la sección 3 se presenta la metodología, con la descripción de la fuente de datos, la construcción de indicadores y el planteo de los métodos estadísticos a usar; en la sección 4 los principales resultados del análisis cuantitativo $y$, por último, en la sección 5 las principales conclusiones del estudio y los próximos pasos en la tarea de investigación.

\section{Marco Teórico}

El presente estudio se enmarca dentro de la economía del conocimiento e innovación, particularmente en la literatura de SI. El marco teórico se nutre con aportes de corte neoschumpeterianos, evolucionistas e institucionalistas, con especial atención en la naturaleza de las actividades innovativas de las empresas. En esta sección presentamos, en el primer apartado el enfoque de SNI, sus distintas vertientes y versiones, así como el planteo del problema que inquieta a esta investigación; mientras que en el segundo apartado presentamos un esquema teórico que se considera pertinente para el abordaje de la problemática, el SNI desde una perspectiva de tramas productivas.

\footnotetext{
2 - Esta sistematización puede profundizarse en Carlsson (2006).
} 


\section{Sistemas de Innovación y Planteo del Problema}

Históricamente el enfoque de $\mathrm{SNI}$ se inició a fines de la década de los ' 80 como marco para estudiar el desempeño económico de los países desde una perspectiva histórica y holística, a partir del trabajo de Freeman (1987) e introducido explícitamente por Lundvall (1988). El enfoque reconoce en la historia del pensamiento económico como antecedentes a List (I84I) y Babbage (I832), y puede decirse que conceptualmente el enfoque de Sistemas de Innovación tiene dos grandes influencias teóricas dentro de la economía de la innovación y el cambio tecnológico: las teorías evolucionistas y las teorías del aprendizaje interactivo (Edquist, 1997).

En primer lugar, este enfoque está influenciado por las teorías evolucionistas de la firma y del cambio tecnológico vinculadas a las ideas neo-schumpeterianas. En esta tradición el cambio tecnológico es un proceso evolutivo que no está guiado por procesos optimizadores, sino por mecanismos rutinizados que desarrollan las empresas. Son estos mecanismos habituales de las firmas los que introducen novedades en el sistema, en función de los procesos sociales de selección existentes de innovaciones (Nelson y Winter 1977, 1982; Nelson, 1987).

En segundo lugar, en enfoque de SNI está enraizado en las teorías del aprendizaje interactivo, más bien vinculadas a la tradición institucionalista en la disciplina. Aquí la tecnología es concebida como un cúmulo de conocimiento y la empresa como un sistema complejo de generación y difusión del mismo (Ernst y Lundvall, 1997). Desde esta perspectiva, se considera que los procesos de innovación se caracterizan, en importante medida por ser procesos de aprendizaje interactivo. Esto es, producto de interacciones tanto al interior de las organizaciones, como a través de interacciones entre diferentes organizaciones o agentes (ya sea entre empresas entre sí 0 entre empresas y otras instituciones).

Conceptualmente, el SNI cuenta con dos dimensiones básicas (Lundvall, 1992). En primer lugar, en tanto la innovación refleja los procesos de aprendizaje y éstos dependen de las actividades rutinarias de las empresas, la innovación está enraizada en la estructura productiva y económica de una nación, pues ésta limita las direcciones y alcances de las rutinas productivas. En segundo lugar, la configuración institucional es la que forma y permite que la actividad innovativa ( $y$ económica en general) tenga lugar en un ambiente caracterizado por la incertidumbre. Por un lado, las instituciones ${ }^{3}$ moldean los hábitos humanos, afectando la forma que adquieren las rutinas productivas $y$, por otro, generan la estabilidad necesaria como para sostener determinadas actividades en el tiempo en contextos de cambio continuo (Johnson, 1992). De este modo, la tasa y la dirección de la innovación, de la que dependen en última instancia el desempeño económico de una nación, depende de la co-evolución entre los distintos aspectos de la configuración institucional y de la estructura económica.

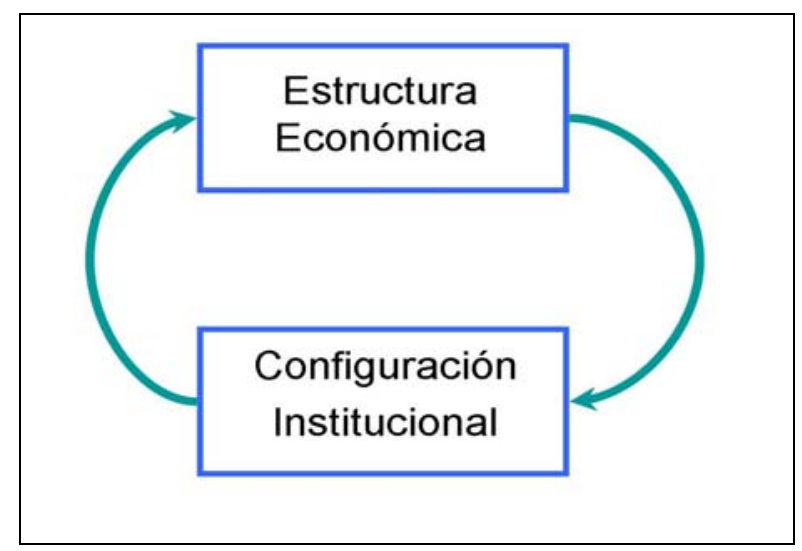

Figura I. Dimensiones del SIN.

Adaptado de Lundvall (I992:8-13) y Johnson (I992:25)

A grandes rasgos, hay dos perspectivas dentro del marco de SNI que enfatizan distintos modos de aprendizaje e innovación. Siguiendo a Jensen, et al. (2007) y Lundvall (2007) hay dos modos ideales de aprendizaje e innovación: el modo STI (Sciencie, Techonology and Innovation) y el modo DUI (Doing, Using and Interacting). El modo de aprendizaje STI se centra en la gestión de conocimiento codificado y técnico (principalmente know-why), sea a través del manejo de laboratorios de I+D o a través de la cooperación con investigadores del sector científico. Se refiere básicamente a cómo las empresas usan y realizan desarrollos sobre conocimiento de tipo científico. El modo de aprendizaje DUI, por su parte, es un modo de aprendizaje basado fundamentalmente en la experiencia diaria $y$ en el desarrollo de interacciones principalmente informales. Así

\footnotetext{
3 - La definición amplia de instituciones es: "conjunto de hábitos, rutinas, reglas, normas y leyes, que regulan las relaciones entre la gente y forman la interacción humana" (Johnson, 1992). Esta definición incluye las instituciones formales, tales como agencias gubernamentales, cámaras empresariales, sindicatos, etc., pero las trasciende.
} 
es que dentro de las perspectivas de SNI hay una visión estrecha, que se focaliza en el modo STI de aprendizaje e innovación, y una perspectiva más amplia que, además de considerar el modo STI, hace especial énfasis en el modo DUI (Lundvall, et al., 2009)

Dentro de la primera línea, el SNI se define como “(...) el conjunto de instituciones cuya interacción determina la performance innovativa de las firmas nacionales" (Nelson y Rosenberg, 1993). Desde esta perspectiva el estudio se centra en las especificidades de las instituciones nacionales y las políticas que directa $o$ indirectamente apoyan la generación y difusión del conocimiento, analizando principalmente la relación entre las instituciones formales de Ciencia y Tecnología (CyT) y el aparato productivo (Nelson y Rosenberg, 1993).

Por su parte, la visión más amplia señala que “(...) un Sistema de Innovación está constituido por elementos y relaciones que interactúan en la producción, difusión y uso de conocimiento nuevo y económicamente útil" (Lundvall, 1992). En ese sentido, un SNI se refiere a elementos y relaciones que están localizadas dentro de una economía nacional. Esta perspectiva toma en cuenta las instituciones sociales, las empresas y su organización interna, la regulación macroeconómica, la configuración institucional del sector financiero $y$ el sistema de I+D, la infraestructura educativa $y$ en comunicación $y$ las condiciones de mercado, en tanto estos aspectos tienen impacto en los procesos de aprendizaje y de construcción de competencias de las empresas.

La definición desde esta perspectiva ha sido ampliada a modo de explicitar el tratamiento del modo STI y DUI de aprendizaje y otros avances, de la siguiente manera: " $E I$ sistema nacional de innovación es un sistema complejo, abierto $y$ en evolución, que abarca las relaciones al interior y entre organizaciones, instituciones y estructuras sociales, que determinan la tasa y dirección de la innovación y de la construcción de competencias que emanan de los procesos de aprendizaje basados tanto en la ciencia, como en la experiencia" (Lundvall, et al., 2009).

Por otra parte, dentro de la literatura han surgido diversas líneas complementarias (Edquist, 200I) de SI según el nivel de análisis. Así, algunos estudios se focalizan en sistemas regionales de innovación (Ohmae, 1993; Ashein y Gertler, 2004), una importante línea de ellos en sistemas sectoriales de innovación (Malerba, 2002, 2004; Breschi y Malerba,
1997), algunos en sistemas locales de innovación (Appendini y Nuijten 2002) o sistemas supranacionales de innovación (Caracostas y Soete, 1997) y otros en sistemas corporativos de innovación (Granstrand, 2000).

Sin embargo, en la literatura ha predominado desde un comienzo el nivel de análisis nacional. Ello en reconocimiento de que la forma en que las empresas adquieren conocimiento está fuertemente condicionada por las características que tiene el contexto en el que se desenvuelven $y$, por tanto, empresas de distintas naciones experimentarán procesos de aprendizaje que diferirán porque, incluso estando inmersas en un sistema económico similar al de otros países, el medio en que operan ( $y$ aprenden) tiene características idiosincrásicas, ideológicas, culturales, económicas e históricas propias (Johnson, 1992). De allí surge, según los autores enrolados en este enfoque, la importancia de lo "Nacional" al evaluar los procesos de aprendizaje, de desempeño empresarial y económico ${ }^{4}$.

Una preocupación recurrente en la literatura de SNI es la manera en que el carácter de nacional de los SI se ve afectado por la internacionalización de la producción. En las últimas décadas las características salientes del contexto económico y tecno-productivo involucran un conjunto de transformaciones que se pueden caracterizar como de internacionalización. Este conjunto de transformaciones engloba: a) una gran expansión de los flujos internacionales de comercio, de capitales, de tecnología y de información; b) una interconexión e interrelación más intensa entre los distintos sistemas productivos y socio-institucionales de las naciones; $y$ c) una simultánea transnacionalización de los distintos agentes económicos, con una mayor importancia de las empresas multinacionales y de la inversión extranjera directa en las economías nacionales (López, 2007). En este estado de cosas, la bibliografía en SNI señala la preocupación acerca de cuál es el efecto que estas últimas transformaciones pueden tener sobre el carácter de "nacional" de los SI, y

\footnotetext{
4 - Además, una de las más importantes aplicaciones de este enfoque es para analizar los procesos de desarrollo económico de los países (López, 2006), en reconocimiento de que la innovación a nivel micro, meso y macro económico es la principal fuerza subyacente al crecimiento de las economías nacionales. El enfoque de SI se ha señalado como un fehaciente instrumento analítico para abordar la problemática del desarrollo, sobre todo por su carácter holístico e interdisciplinario (Lundvall, et al., 2009).
}

ISSN: 07I 8-2724. (http://www.jotmi.org) 
en particular, sobre la relevancia que en este contexto tienen las fuentes domésticas de obtención de conocimiento para la innovación. (Lundvall, 1992; Johnson, 1992; Nelson, 1993; López, 1996; Chudnosky, 1999; Balzat y Hanusch 2004; Lundvall, 2007).

El objetivo general de esta investigación es estudiar la capacidad de los SNI de continuar afectando la actividad innovadora en ambientes altamente internacionalizados. Dentro de esta problemática general, interesa específicamente estudiar si en actividades productivas internacionalizadas de una economía en desarrollo, que se encuentra alejada de la frontera tecnológica internacional, las fuentes nacionales de obtención de conocimiento mantienen cierta relevancia para la performance innovativa de las firmas o si, por el contrario, su importancia es marginal. Esto implica investigar si en contextos de alta internacionalización tiene relevancia el conocimiento creado por los agentes locales, al interior de sus organizaciones y por la interacción con otros actores nacionales. Para el abordaje de esta problemática, se plantea un enfoque de SNI combinado con un enfoque de tramas productivas.

\section{SNI desde una Perspectiva de Tramas Productivas}

Una trama productiva es un espacio económico de construcción de capacidades tecnológicas y competencias que está conformado, por un lado, por una o más empresas organizadoras (el o los núcleos de la trama) y el conjunto de sus proveedores y clientes ${ }^{5}$; y por otro, por sus interrelaciones derivadas de las compras y ventas y los flujos de información y conocimiento comercial $y$ productivo, a través de canales formales e informales (Albornoz, Milesi y Yoguel, 2005; Novick y Carrillo, 2006). Una trama productiva es una comunidad epistémica (Cowan, David y Foray, 2000) que comparte un lenguaje codificado y desarticulado, y el conocimiento involucrado es difícil de ser entendido por agentes que no pertenecen a la trama.

De este modo, dentro de este marco conceptual las tramas productivas se definen en paralelismo a la concepción de Sls, como elementos (actores) y sus

\footnotetext{
5 - En ese sentido, trasciende a la definición de sector, puesto que todos los proveedores del núcleo, no necesariamente van a pertenecer a su mismo sector y componentes de un mismo sector pueden pertenecer a distintas tramas productivas.
}

interrelaciones. Sin embargo, también se presenta como una noción que se focaliza en analizar interrelaciones más definidas, en el sentido de concentrarse en actores que comparten un lenguaje específico $y$, por tanto, de una base común de conocimiento.

Una trama productiva involucra flujos de bienes y servicios en una relación estable de largo plazo. La trama se instituye como un contexto donde las empresas se desenvuelven y que les permite, a través de la interacción, especificar el conocimiento codificado y externalizar el conocimiento tácito (Novick y Carrillo, 2006), facilitando el intercambio y la acumulación colectiva de estos tipos de conocimiento. De este modo, a través de la interacción, se dan diversos procesos de generación, circulación, apropiación de conocimiento y de sinergias en los procesos de aprendizaje.

En una trama productiva los procesos de aprendizaje y el desarrollo de capacidades tecnológicas dependen de: a) las competencias endógenas (conocimiento acumulado que permite la absorción futura de nuevo conocimiento y afecta la utilidad del conocimiento adquirido) acumuladas a lo largo del tiempo, producto de procesos internos de aprendizaje interactivo; y b) los flujos de conocimiento vía la interacción con otros agentes, lo que incluye tanto vinculaciones al interior de la trama (entre los proveedores y clientes con el núcleo, y entre las empresas de la trama entre sí), como vinculaciones fuera de la trama (entre empresas de la trama e instituciones, cooperación formal e informal, vinculaciones con instituciones técnicas, etc.) (Albornoz, Milesi y Yoguel, 2005).

Como nos interesa el efecto que la internacionalización tiene sobre los modos de aprendizaje interactivo de las empresas, es necesario distinguir entre tramas productivas internacionalizadas y no internacionalizadas. De este modo, y según representamos en la Figura 2, tramas internacionalizadas son aquellas que tienen importantes interacciones con el resto del mundo, tanto de índole productiva, así como flujos de información y de conocimiento (tengan o no núcleo dentro del país). Por su parte, una trama no internacionalizada es aquella que mantiene escasas o nulas vinculaciones productivas, de información y/o conocimiento, con el resto del mundo. 


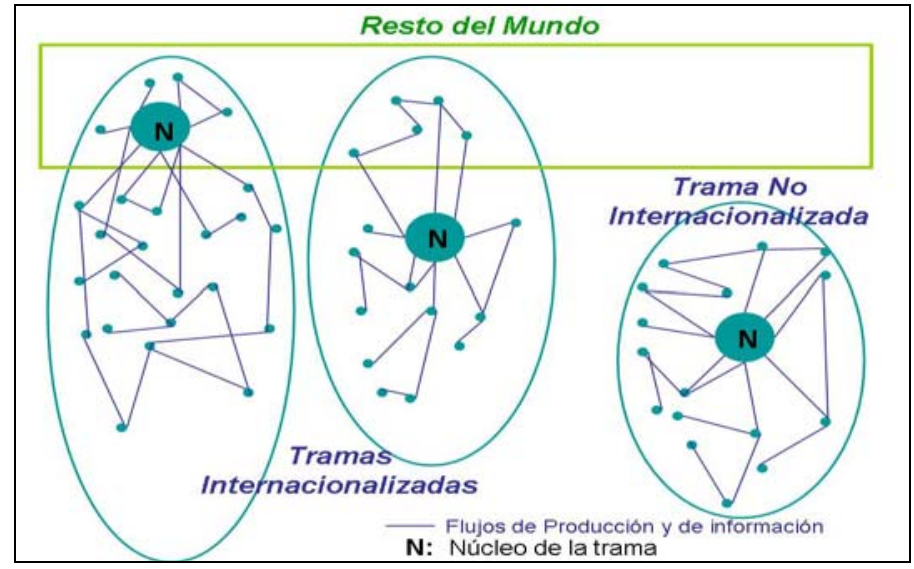

Figura 2.: Tramas Productivas Internacionalizadas

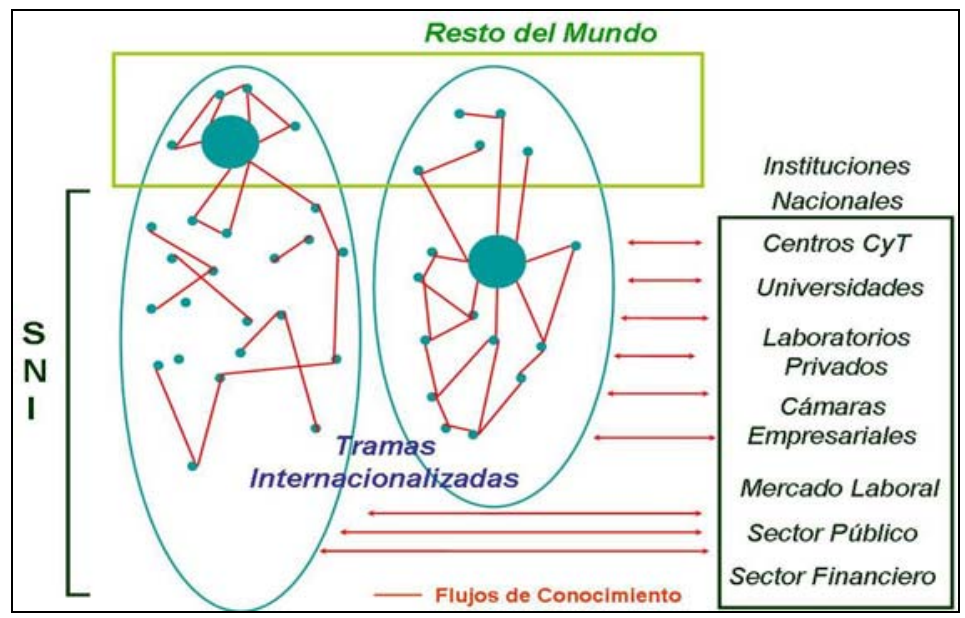

Figura 3. SNI desde una perspectiva de tramas productivas.

En la Figura 3 presentamos una representación simplificada de un SNI desde una perspectiva de tramas productivas $^{6}$. Siguiendo la definición de Lundvall (1992) está conformado por elementos y sus interrelaciones. Los elementos son, por un lado las empresas de las tramas productivas (núcleos, sus proveedores y clientes) teniendo en cuenta los procesos internos de aprendizaje interactivo y sus competencias acumuladas, y por otro, las instituciones, tales como los centros públicos de СyТ,

6 - Esta representación responde al principal interés de la investigación, que es estudiar la relevancia del SNI para tramas productivas internacionalizadas. Sin embargo, es posible ampliar el enfoque e incluir tramas productivas no, o escasamente, internacionalizadas atendiendo a la Figura 2 presentada anteriormente.
Universidades, los laboratorios privados, la organización de los mercados laborales, las cámaras empresariales, el sector público y características del sector financiero. Por último, el sistema está conformado por las interrelaciones, que son los flujos de conocimiento entre los elementos, como vinculaciones tecnológicas y comerciales relevantes para la actividad innovativa $y$ procesos de aprendizaje de las empresas.

Entonces, dentro de este marco de SNI desde una perspectiva de tramas, el objetivo particular de esta investigación es analizar la importancia que tiene el SNI, para empresas locales que se desenvuelven en tramas productivas sumamente internacionalizadas en un país en desarrollo, como es el caso de la Argentina. 


\section{Metodología}

En el abordaje de esta problemática, y a los fines de este artículo, se ha seleccionado una trama productiva internacionalizada argentina, que es la trama automotriz ${ }^{7}$. En un país en desarrollo, como la Argentina, esta trama organiza su producción alrededor de empresas que son filiales de empresas multinacionales con casa matriz en otros países ${ }^{8}$. Por lo que los núcleos de la trama global se encuentran fuera de la economía nacional $y$, fronteras adentro, la producción se organiza alrededor de las terminales de empresas multinacionales en el país, en tanto que los encadenamientos se concentran fundamentalmente hacia atrás desde las mismas (proveedores de insumos, producción de auto-partes y provisión de servicios).

El estudio se basa en datos provenientes de un relevamiento efectuado a 89 empresas productoras de auto-partes de Capital Federal y Gran Buenos Aires, Córdoba y Rafaela a lo largo del 2006. Dos tercios de las empresas relevadas son de capital nacional, predominando las firmas con ventas superiores a los 5 millones de dólares anuales y con más de 50 ocupados. En la muestra están sobre-representados los proveedores directos o indirectos de las terminales automotrices. El $79 \%$ de las empresas encuestadas pertenece a dicho grupo, lo que implica que la muestra incluye a poco más del $25 \%$ del total de empresas fabricantes de partes $y$ componentes para la industria automotriz con planta productiva en Argentina. Por su parte, las empresas que venden casi exclusivamente al mercado de reposición representan el $21 \%$ restante de la muestra y algo menos del $5 \%$ del total de empresas autopartistas argentinas que destinan su producción principalmente a dicho mercado.

\footnotetext{
7 - Este artículo forma parte de una investigación más amplia, en el marco del plan de trabajo de la beca doctoral (CONICET) "Procesos de generación de conocimiento en tramas productivas internacionalizadas y Sistema Nacional de Innovación". Esta investigación también contempla el estudio de otra trama productiva internacionalizada, a modo de comparar el efecto del SNI en tramas con distinto tipo de internacionalización. Para profundizar acerca de las principales características de la trama automotriz en la Argentina, puede consultarse Motta y Barbero (2007).

8 - Aunque no es la regla para absolutamente todas las economías en desarrollo, habiendo casos de estos países, como el de India, que cuentan con grandes productores finales de vehículos de base nacional.
}

La encuesta relevó información sobre cuestiones estructurales generales de las empresas (tamaño, propiedad del capital, exportaciones, ventas, empleo, etc.), sobre la estructura de compra - ventas en la trama productiva (distribución de las ventas, compras, proveedores de materias primas y partes, etc.), vinculaciones y relaciones con los agentes de la trama (relaciones contractuales, actividades de cooperación, vinculaciones técnicas, etc.), actividades innovativas (tipos de innovación introducidas, resultados, importancia de las innovaciones, gastos en actividades innovativas, distribución del personal, calidad, etc.), gestión de los recursos humanos (modos de organización del proceso de trabajo, contratación, etc.) y actividades de capacitación. Con esta información se construyó una serie de indicadores relevantes para comprender los procesos de aprendizaje de las empresas: indicadores de Aprendizaje Interactivo Interno, Compra Externa de Tecnología, de Vinculaciones y de Desempeño Innovador. Estos indicadores serán usados en un Análisis Factorial de Correspondencias Múltiples (AFCM), a modo de poder relacionar distintos modos de aprendizaje de las empresas, con su performance innovativa.

Para esta labor, se diseñó en primer lugar un indicador global de aprendizaje interactivo interno (AI) que procura captar el grado de virtuosidad de los procesos de circulación de conocimiento al interior de la empresa. Este indicador está construido sobre la base de dos subindicadores igualmente ponderados: el indicador de estructura de circulación interna de conocimiento (ECC) y el indicador de Intensidad de Circulación de Conocimiento (ICC). El primer sub-indicador trata de captar los aspectos vinculados a la estructura de las firmas que condicionan la circulación del conocimiento al interior de las empresas y los procesos de aprendizaje. Por eso resume distintos aspectos vinculados a la organización del proceso de trabajo (el trabajo en equipo, las modalidades de supervisión y el grado de autonomía dado al trabajador, así como su rotación), la estructura de I+D y la estructura de capacitación?. El segundo sub-

9 - Todos estos sub-indicadores tienen tres modalidades: bajo, medio y alto, en función de la medida en que favorecen los procesos de circulación de conocimiento. El indicador de Organización de Trabajo resume tres sub-indicadores: de Trabajo en equipo (según si la modalidad de trabajo es individual, por células y con distribución de tareas, y por células con tareas de programación y puesta y mantenimiento de primer nivel), de Adquisición de experiencia (considerando las 
indicador procura captar los aspectos del aprendizaje interno de la empresa asociados a las actividades de innovación y de aprendizaje. Entonces incluye los esfuerzos en desarrollo interno de tecnología (si se realizan gastos en I+D, desarrollo de productos, cambio organizacional, etc.), las actividades de calidad y las de mejora continua ${ }^{10}$.

Las empresas también realizan esfuerzos que involucran la compra externa de tecnología. Para captar este fenómeno se construyó un indicador que mide la intensidad de esta fuente de conocimiento según si la firma ha realizado gastos en la compra de bienes de capital y/o en la adquisición de licencias.

modalidades de trabajo el grado y planificación de la rotación), y de Autonomía de los empleados y Rol del Supervisor sobre la circulación de conocimiento entre niveles de la empresa (considerando las competencias requeridas a los operarios y las funciones que cumplen los líderes y supervisores en el proceso de trabajo: si está vinculado a formas de control, si tiene una intervención limitada o tiene un rol integrador. El indicador de Estructura en Capacitación se construye atendiendo a la posibilidad de circulación de conocimiento vinculado a actividades de capacitación, según la existencia de estructura para capacitación y el desarrollo de la función; y el indicador de Estructura en I+D, se construye análogamente, respecto a las actividades de investigación y desarrollo, según el establecimiento de funciones y estructura para este tipo de actividades.

10 - Todos estos sub-indicadores tienen tres modalidades: bajo, medio y alto, en función de la medida de la intensidad de los procesos de circulación de conocimiento y actividades de innovación. El indicador de Cultura hacia la calidad considera la existencia y desarrollo de actividades vinculadas a la cultura hacia la calidad: comunicaciones periódicas con clientes, existencia de política de calidad comunicada al personal, definición y exposición publica de indicadores clave, existencia de equipos de solución de problemas, existencia de auditoría interna, sistema de mantención del orden y limpieza, y sistema de reconocimiento de ideas. El Indicador de Mejora Continua, tiene en cuenta, por un lado, el grado de aplicación de diferentes ítems referidos al control de productos y/o procesos (definición de requisitos del producto, control de características críticas del producto, documentación de actividades críticas del proceso, controles de proceso, registro de datos, archivo de datos, especificación de materias primas e insumos críticos, existencia de rastreabilidad y existencia de rutina de calibración) y por otro, la utilización de métodos sistemáticos para mejora e innovación y el nivel de participación de los ocupados en la utilización de las mismas. Por último, el indicador de Desarrollo interno de tecnología considera la cantidad de actividades en que la empresa ha realizado gastos en desarrollos propios: en I+D, programas de mejora continua, desarrollo de productos, cambio organizacional y comercial, y capacitación orientada a la innovación.
Por otro lado, se han construido indicadores de Vinculaciones, según se representa en la figura 5, tratando de diferenciar las vinculaciones con agentes nacionales de aquellas con agentes internacionales. La intensidad de las vinculaciones es considerada a través de tres factores: la cantidad de objetivos de la vinculación" ", la frecuencia de las interacciones (semanal, diaria, etc.) ${ }^{12}$, y la cantidad de agentes según se trate de vinculaciones nacionales (terminales, plantas, proveedores o clientes nacionales, cámaras, centros tecnológicos o universidades) o internacionales (plantas, clientes o proveedores internacionales, o casa matriz de empresas extranjeras).

11 - Para construir los indicadores de vinculaciones por objetivos, se ponderan los objetivos de las vinculaciones (conseguir clientes, proveedores, contratar empleados, información sobre el contexto de negocios, información tecnológica, desarrollo de productos, de exportaciones, capacitación, financiamiento, desarrollo de redes de circulación de información y de espacios de intercambio de conocimiento útil para su empresa) para todos los agentes (Plantas Nacionales, Plantas Internacionales, Terminales, Casa Matriz, Proveedores Nacionales, Proveedores Internacionales, Clientes Nacionales, Clientes Internacionales, Cámaras, Consultores, Centros Tecnológicos, Universidades), y según el puntaje ponderado se establecen las modalidades Inexistente, Bajo, Medio y Alto. Luego sumando los indicadores de todos los agentes se consolida un indicador global de Vinculaciones por Objetivo, con modalidades Bajas, Medias y Altas, un indicador global de Vinculaciones Nacionales por Objetivo (promediando los indicadores por objetivos de Plantas nacionales, Clientes Nacionales, Proveedores Nacionales, Terminales, Centros Tecnológicos y Universidades), y un indicador global de Vinculaciones Internacionales por Objetivo (promediando análogamente los indicadores por objetivos de los agentes Plantas internacionales, Clientes internacionales, Proveedores internacionales y Casa Matriz).

12 - Para construir estos indicadores, según la frecuencia de las vinculaciones, se construyen las modalidades Inexistente, Baja (esporádica), Media (trimestral o mensual) y Alta (semanal o diaria), para todos los agentes. Análogamente a los indicadores globales por objetivos, se construyen un indicador global de Vinculaciones por Frecuencia, de Vinculaciones Nacionales por Frecuencia $y$ de Vinculaciones Internacionales por Frecuencia. 


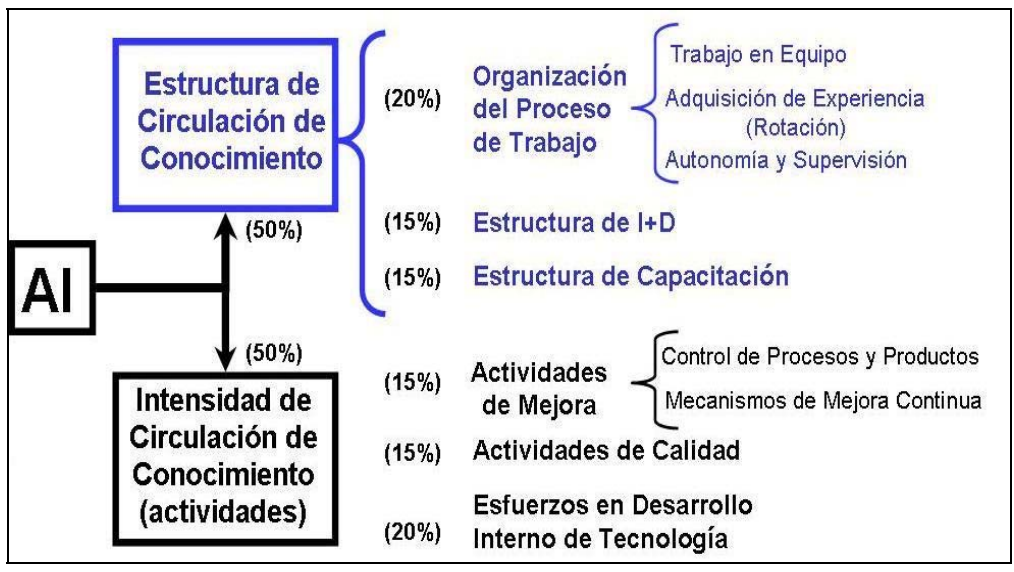

Figura 4. Construcción del Indicador de Aprendizaje Interno.

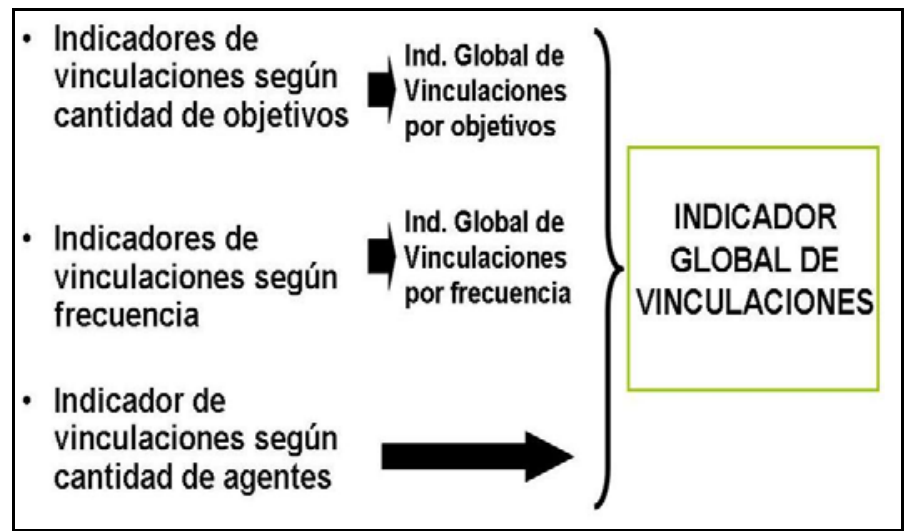

Figura 5. Construcción de Indicadores de Vinculaciones.

Por último, se ha desarrollado un indicador global del desempeño innovador de las firmas teniendo en cuenta las de áreas o tipos de innovaciones realizadas (en productos, procesos, en comercialización y en materia organizacional), la importancia de las innovaciones introducidas y los resultados de las mismas (mejoras en la eficiencia de los $\mathrm{RRHH}$, en el JIT interno, en el desarrollo y mejora de productos, en la adaptación de productos, desarrollo y mejora de procesos, en el desarrollo de nuevas formas de distribución y en cambios en las formas de producción). Teniendo en cuenta la cantidad de áreas donde se obtuvieron resultados en innovación y la importancia global de las innovaciones, se ha desarrollado un indicador global del desempeño innovador de las firmas.

A fines ilustrativos, y de utilidad en la presentación de los resultados en la sección siguiente, en la Tabla I resumimos los valores muestrales de los indicadores clave del análisis. Allí puede apreciarse que para alrededor del $50 \%$ de las empresas de la muestra el aprendizaje interactivo interno y las vinculaciones con agentes nacionales obtienen la modalidad alta, en tanto que las vinculaciones con agentes externos (19\%) y la compra externa de tecnología (33\%) tiene una modalidad alta para un porcentaje significativamente menor de empresas. Sin embargo, esto por sí solo no implica una mayor importancia relativa de las fuentes internas de conocimiento respecto de las externas para el desempeño innovador de las empresas. Ello porque: a) no necesariamente van a coincidir las empresas que tengan alto aprendizaje interno con las que tengan altas vinculaciones nacionales, pudiendo darse distintas combinaciones de importancia relativa de las fuentes internas y externas en los procesos de aprendizaje de las empresas, y b) si esto ocurriera, no necesariamente 
debieran coincidir las que tienen alto aprendizaje interno y vinculaciones nacionales con las empresas relativamente más innovadoras de la muestra, pudiendo en éstas darse otra importancia de las fuentes externas e internas. Por todo ello, es necesario avanzar en el análisis estadístico con técnicas que nos permitan superar estas limitaciones.

\begin{tabular}{|l|ccc|}
\hline & Bajo & Medio & Alto \\
\hline Aprendizaje Interactivo Interno & $17,98 \%$ & $25,84 \%$ & $56,18 \%$ \\
ECC & $15,73 \%$ & $41,57 \%$ & $42,70 \%$ \\
ICC & $14,61 \%$ & $38,20 \%$ & $47,19 \%$ \\
Compra Externa de tecnología & $20,22 \%$ & $43,82 \%$ & $33,71 \%$ \\
Vinculaciones & $38,20 \%$ & $25,84 \%$ & $35,96 \%$ \\
Vinculaciones Nacionales & $21,35 \%$ & $34,83 \%$ & $43,82 \%$ \\
Vinculaciones Internacionales & $47,19 \%$ & $33,71 \%$ & $19,10 \%$ \\
Indicador de Innovación & $37,08 \%$ & $22,47 \%$ & $39,33 \%$ \\
\hline
\end{tabular}

Tabla I. Valores Muestrales de los Indicadores Clave.

Como todos los indicadores definidos son de naturaleza cualitativa, para su análisis cuantitativo es necesario aplicar técnicas de manejo multivariante de datos. Así, se utiliza el método de AFCM a modo de reducir dimensiones entre categorías diversas de variables cualitativas y poder caracterizar los procesos de aprendizaje e identificar las principales fuentes de conocimiento para las empresas de esta trama.

Luego aplicando técnicas de Cluster, elaboramos tipologías de empresas según modos de acumulación y adquisición de conocimiento, y se forman grupos homogéneos de empresas en función de los principales modos de aprendizaje. Este análisis permite obtener una primera aproximación a la importancia que reviste el SNI en el proceso de acumulación de conocimiento de esta trama, diferenciando la importancia relativa que tienen las fuentes externas e internas de obtención de conocimiento de las empresas y mostrando la relación existente entre éstas con el desempeño innovador de las empresas.

La hipótesis general que guía el trabajo empírico es que las empresas que complementan más sus fuentes externas de conocimiento con conocimiento doméstico, tendrán un desempeño innovador y competitivo mejor que aquellas firmas cuyas fuentes de conocimiento son casi exclusivamente externas.

\section{Resultados}

A los fines de poder captar cuantitativamente la complejidad de los aspectos que hacen a los procesos internos de aprendizaje $y$ de aprendizaje a través de interacción con agentes externos a la empresa, se redujeron dimensiones de las modalidades del indicador global de vinculaciones $y$ del indicador global de aprendizaje interactivo interno (variables activas del AFCM). De este modo, se construyeron 4 factores que acumulan el $100 \%$ de la inercia (ver Figura 6), y se proyectaron sobre estas dimensiones como suplementarias el resto de las variables relevantes, con especial atención en las variables de innovación. La principal meta de este análisis es poder construir grupos de empresas relativamente homogéneas respecto a los principales rasgos de sus procesos de aprendizaje (la importancia relativa de los componentes externos de conocimiento $y$ de las fuentes domésticas o nacionales e internas a la firma), y evaluar luego su desempeño innovador (aspecto sumamente heterogéneo) en dimensiones homogéneas que permitan el cálculo de distancia entre las empresas. 


\begin{tabular}{|c|c|c|c|c|}
\hline \multicolumn{5}{|c|}{ HISTOGRAMME DES 4 PREMIERES VALEURS PROPRES } \\
\hline NUMERO & $\begin{array}{l}\text { VALEUR } \\
\text { PROPRE }\end{array}$ & POURCENT. & $\begin{array}{c}\text { POURCENT. } \\
\text { CUMULE }\end{array}$ & \\
\hline 1 & 0.6394 & 31.97 & 31.97 & 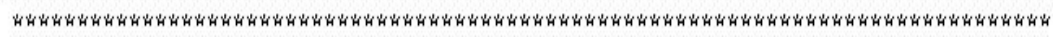 \\
\hline$\frac{1}{2}$ & 0.5627 & 28.13 & 60.10 & 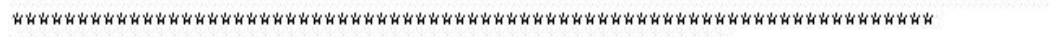 \\
\hline 3 & 0.4373 & 21.87 & 81.97 & 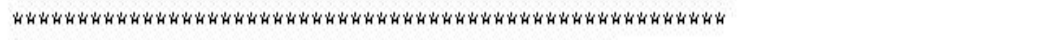 \\
\hline 4 & 0.3606 & 18.03 & 100.00 & 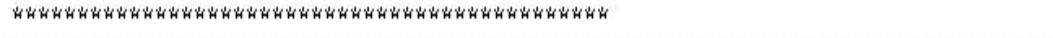 \\
\hline
\end{tabular}

Figura 6. Histograma de los valores propios.

De este modo, luego del AFCM, se aplicaron técnicas de cluster y se construyeron 5 grupos de empresas según las variables activas (el corte puede verificarse en la Figura 7 a continuación), y se comprobó un desempeño innovador diferencial acorde a los distintos modos de aprendizaje predominantes en cada grupo, como se presenta a continuación.

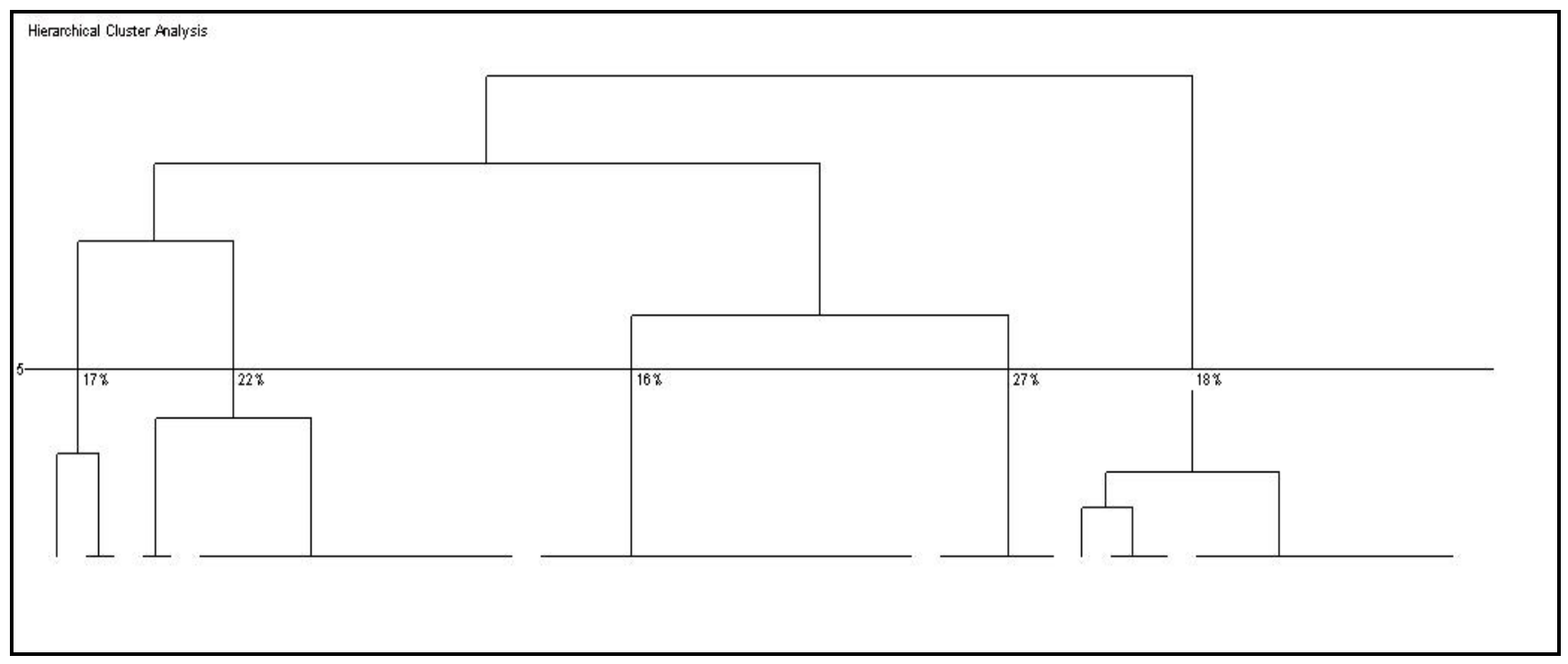

Figura 7. Corte de Cluster.

El grupo I abarca al $27 \%$ de las empresas de la muestra y presenta un alto aprendizaje interactivo interno $y$ altas vinculaciones. Aquí están sobre representadas las empresas que cuentan con una alta capacidad estructural de aprendizaje (el $75 \%$ tiene una estructura de circulación de conocimiento virtuosa) y que tienen intensas actividades de aprendizaje (el $79 \%$ de las empresas tiene un alto indicador de intensidad), configurando un grupo donde la totalidad de las empresas que lo integran tienen un alto indicador de aprendizaje interactivo. Por su parte, están sobre representadas las empresas que recurren en término medio a la compra externa de tecnología. Respecto a las vinculaciones, están sobre representadas las empresas que tienen altas vinculaciones con agentes nacionales $(87,5 \%$ del grupo tiene altas y ninguna empresa tiene bajas), en tanto que están sobre representadas las empresas que tienen medias vinculaciones con agentes internacionales. En suma este es un grupo que balancea sus fuentes de conocimiento, con un alto aprendizaje interno, complementado con compra externa de tecnología y con altas vinculaciones, mayormente con agentes nacionales, pero también con agentes extranjeros. En este grupo predominan las empresas que tienen una alta introducción de innovaciones y de importancia, alcanzando los dos tercios del grupo la modalidad alta del indicador global de innovación. Además están sobre representadas en este grupo las empresas que han introducido innovaciones de todos los tipos: en materia de procesos (cerca del $92 \%$ de las empresas), de tipo comercial (54\%), organizacional (79\% del grupo) y en el desarrollo y mejora de productos (87,5\%). 


\begin{tabular}{|c|c|c|c|c|}
\hline Variable & $\begin{array}{l}\text { Modalida } \\
\text { d }\end{array}$ & $\begin{array}{l}\% \text { de la modalidad } \\
\text { en el grupo }\end{array}$ & $\begin{array}{c}\% \text { de la } \\
\text { modalidad en la } \\
\text { muestra }\end{array}$ & Sign. \\
\hline Aprendizaje interativo & Alto & 100,00 & 56,18 & * \\
\hline Estructura CC & Alta & 75,00 & 42,70 & * \\
\hline Intensidad CC & Alta & 79,17 & 47,19 & * \\
\hline Compra externa de tecnología & Media & 58,33 & 43,82 & $* * * *$ \\
\hline Vinculaciones & Alta & 100,00 & 38,20 & * \\
\hline Vinculaciones Nacionales & Alta & 87,50 & 43,82 & $*$ \\
\hline Vinc nacionales por agentes & Alta & 83,33 & 42,70 & $*$ \\
\hline Vinc nacionales por frecuencia & Alta & 66,67 & 32,58 & $*$ \\
\hline Vinc nacionales por objetivo & Alta & 16,67 & 6,74 & ** \\
\hline Vinc nacionales por objetivo & Media & 83,33 & 65,17 & ** \\
\hline Vinculaciones Internacionales & Media & 50,00 & $33,7 \mid$ & $* *$ \\
\hline Vinc internacionales por objetivo & Media & 70,83 & 41,57 & $*$ \\
\hline Vinc internacionales por agentes & Media & 50,00 & $33,7 \mid$ & ** \\
\hline Vinc internacionales por frecuencia & Alta & 29,17 & 16,85 & $* * *$ \\
\hline Indicador de Innovación & Alto & 66,67 & 39,33 & $*$ \\
\hline Innovación de Tipo Comercial & Sí & 54,17 & 30,34 & * \\
\hline Innovación de Tipo Organizacional & Sí & 79,17 & 48,31 & $*$ \\
\hline Innovación de Tipo Productos & Sí & 87,50 & 74,16 & $* * *$ \\
\hline Innovación de Tipo Procesos & Sí & 91,67 & 75,28 & $* *$ \\
\hline \multicolumn{4}{|c|}{ Variables Subrepresentadas } & \multirow{3}{*}{$\begin{array}{l}* * \\
*\end{array}$} \\
\hline Intensidad CC & Baja & 0,00 & $|4,6|$ & \\
\hline Estructura CC & Baja & 0,00 & 15,73 & \\
\hline Compra externa de tecnología & Baja & 4,17 & 20,22 & ** \\
\hline Vinculaciones nacionales & Media & 12,50 & 34,83 & $*$ \\
\hline Vinculaciones nacionales & Baja & 0,00 & 21,35 & $*$ \\
\hline \multirow[t]{2}{*}{ Indicador de Innovación } & Bajo & 12,50 & 37,08 & $*$ \\
\hline & & & & $*$ \\
\hline
\end{tabular}

(') * Significativa al 1\%; ** Significativa al 5\%; *** Significativa al 10\%

Tabla 2. Principales Indicadores Sobre y Sub representados: Grupo I.

El grupo 2, por su parte, representa el $16 \%$ de las empresas de la muestra y manifiesta, al igual que el grupo anterior, un alto aprendizaje interno, pero bajas vinculaciones con otros actores. La totalidad de estas empresas tienen un indicador global de aprendizaje interno alto y están sobre representadas las empresas con una alta intensidad de circulación de conocimiento (87,5\%), aunque presentan una estructura de circulación del mismo entre media y alta. Todas las empresas de este grupo tienen un indicador global de vinculaciones bajo, estando sobre representadas tanto las empresas con bajas vinculaciones nacionales (57\%), como aquellas que mantienen bajas vinculaciones internacionales (7I\%). En este grupo las empresas recurren con intensidad a compra externa de tecnología (alto en 57\%), complementan fuertemente esta fuente con esfuerzos internos de aprendizaje, pero descuidan los flujos externos de conocimiento y los procesos de aprendizaje a través de interacciones con otros actores sociales. En consecuencia, el desempeño innovador de estas empresas es menor que en el grupo anterior, que balancea más sus fuentes de conocimiento, $y$ el indicador de innovación se mantiene en niveles que no se diferencian significativamente de las proporciones muestrales, aunque se destacan en este grupo empresas que han introducido innovaciones de tipo organizacional. 


\begin{tabular}{|c|c|c|c|c|}
\hline Variable & $\begin{array}{l}\text { Modalida } \\
\text { d }\end{array}$ & $\begin{array}{l}\% \text { de la modalidad } \\
\text { en el grupo }\end{array}$ & $\begin{array}{l}\% \text { de la modalidad } \\
\text { en la muestra }\end{array}$ & Sign. ' \\
\hline Aprendizaje interativo & Alto & 100,00 & 56,18 & * \\
\hline Intensidad CC & Alta & 85,71 & 47,19 & $*$ \\
\hline Compra externa de tecnología & Alta & 57,14 & 33,71 & $* *$ \\
\hline Vinculaciones & Baja & 100,00 & 35,96 & * \\
\hline Vinculaciones nacionales & Baja & 57,14 & 21,35 & * \\
\hline Vinc nacionales por objetivo & Baja & 64,29 & 26,97 & $*$ \\
\hline Vinc nacionales por agentes & Baja & 50,00 & 17,98 & * \\
\hline Vinc nacionales por frecuencia & Baja & 42,86 & 15,73 & $*$ \\
\hline Vinculaciones internacionales & Baja & 71,43 & 47,19 & ** \\
\hline Vinc internacionales por objetivo & Baja & 85,71 & 50,56 & $*$ \\
\hline Vinc internacionales por frecuencia & Baja & 64,29 & $33,7 \mid$ & ** \\
\hline Innovación de Tipo Organizacional & Sí & 78,57 & 48,31 & $* *$ \\
\hline \multicolumn{5}{|c|}{ Variables Subrepresentadas } \\
\hline Vinc internacionales por frecuencia & Media & 28,57 & 48,31 & $* * *$ \\
\hline Intensidad CC & Baja & 0,00 & $|4,6|$ & **** \\
\hline Estructura CC & Baja & 0,00 & 15,73 & $* * *$ \\
\hline Intensidad CC & Media & 14,29 & 38,20 & *** \\
\hline Vinculaciones nacionales & Alta & 7,14 & 43,82 & * \\
\hline
\end{tabular}

(') * Significativa al 1\%; ** Significativa al 5\%; *** Significativa al 10\%

Tabla 3. Principales Indicadores Sobre y Sub representados: Grupo 2.

\begin{tabular}{|c|c|c|c|c|}
\hline Variable & $\begin{array}{l}\text { Modalida } \\
\text { d }\end{array}$ & $\begin{array}{c}\text { \% de la } \\
\text { modalidad en el } \\
\text { grupo }\end{array}$ & $\begin{array}{c}\% \text { de la modalidad } \\
\text { en la muestra }\end{array}$ & Sign. ' \\
\hline Aprendizaje interativo & Medio & 40,00 & 25,84 & ***** \\
\hline Intensidad CC & Media & 60,00 & 38,20 & $* *$ \\
\hline Vinculaciones & Media & 100,00 & 25,84 & * \\
\hline Vinculaciones Nacionales & Media & 70,00 & 34,83 & $*$ \\
\hline Vinc nacionales por agentes & Media & 75,00 & 39,33 & $*$ \\
\hline Vinc nacionales por frecuencia & Media & 80,00 & 50,56 & $*$ \\
\hline Vinc internacionales por frecuencia & Media & 65,00 & 48,31 & $* * *$ \\
\hline Vinc nacionales por objetivo & Media & 80,00 & 65,17 & $* * *$ \\
\hline Innovación de Tipo Organizacional & No & 65,00 & 48,31 & **** \\
\hline \multicolumn{5}{|c|}{ Variables Subrepresentadas } \\
\hline Aprendizaje interativo & Bajo & 0,00 & 17,98 & $* *$ \\
\hline Estructura CC & Baja & 0,00 & 15,73 & $* *$ \\
\hline Intensidad CC & Baja & 0,00 & $|4,6|$ & $* *$ \\
\hline Vinculaciones nacionales & Baja & 0,00 & 21,35 & $*$ \\
\hline
\end{tabular}

(') * Significativa al I\%; ** Significativa al 5\%; *** Significativa al 10\%

Tabla 4. Principales Indicadores Sobre y Sub representados: Grupo 3. 


\begin{tabular}{|c|c|c|c|c|}
\hline Variable & $\begin{array}{l}\text { Modalida } \\
\text { d }\end{array}$ & $\begin{array}{c}\% \text { de la modalidad } \\
\text { en el grupo }\end{array}$ & $\begin{array}{c}\% \text { de la } \\
\text { modalidad en la } \\
\text { muestra }\end{array}$ & Sign. I \\
\hline Aprendizaje interativo & Medio & 100,00 & 25,84 & * \\
\hline Intensidad CC & Media & 60,00 & 38,20 & $* * *$ \\
\hline Estructura CC & Media & 60,00 & 41,57 & $* * *$ \\
\hline \multicolumn{5}{|c|}{ Variables Subrepresentadas } \\
\hline Vinculaciones & Media & 0,00 & 25,84 & * \\
\hline Vinculaciones internacionales & Media & 13,33 & 33,71 & \multirow{4}{*}{$\begin{array}{l}* * * \\
* * \\
* * * \\
* * *\end{array}$} \\
\hline Vinc internacionales por agentes & Media & 6,67 & 33,71 & \\
\hline Indicador de Innovación & Alto & 20,00 & 39,33 & \\
\hline Innovaciones Tipo Organizacional & Sí & 26,67 & 48,31 & \\
\hline
\end{tabular}

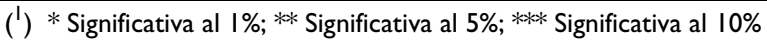

Tabla 5. Principales Indicadores Sobre y Sub representados: Grupo 4.

Luego, en el grupo 3, que abarca el $23 \%$ de la muestra sobresalen las empresas con un aprendizaje interactivo interno medio y con vinculaciones de modalidad media. Así, es que están sobre representadas las firmas con un indicador global de aprendizaje interno medio, con una estructura de circulación de conocimiento entre media y alta y una intensidad de circulación media (60\%). Respecto a las fuentes externas de conocimiento, en este grupo se recurre a compra externa de tecnología en niveles que no se diferencian significativamente de los valores muestrales (ver Tabla I), y en materia de vinculaciones, presenta medias interacciones con agentes nacionales (70\%). Al igual que el grupo anterior, este grupo no se diferencia significativamente de los valores muestrales en materia de innovación.

Por su parte, el grupo 4 engloba al 17\% de las empresas de la muestra, y está compuesto por firmas con un aprendizaje interno medio ( $100 \%$ del grupo) y que mantienen vinculaciones en niveles no significativamente distintos de las medias muestrales (ver Tabla I). El desempeño innovador de las empresas de este grupo es entre medio y bajo, dado que están sub-representadas las empresas con alta Innovación (representando un quinto del grupo). Adicionalmente, están sub-representadas las empresas que han introducido innovaciones de tipo organizacional, que en la muestra representan alrededor del $48 \%$ y en este grupo alcanzan el $26 \%$.
Por último, en el grupo 5, que representa el $18 \%$ de la muestra, predominan las empresas con un bajo aprendizaje interno y con bajas vinculaciones. Cerca del $69 \%$ de las empresas de este grupo cuenta con una deficiente estructura de circulación de conocimiento y más del $62 \%$ presenta una escasa intensidad en actividades de circulación de conocimiento, configurando un grupo donde la totalidad tiene un bajo indicador global de aprendizaje interno. También están sobre representadas las empresas con una baja compra externa de tecnología. Con respecto a las vinculaciones, están sobre representadas las bajas vinculaciones nacionales $(43,75 \%)$, en tanto que las empresas de este grupo mantienen vinculaciones con agentes extranjeros en niveles no significativamente distintos de las medias muestrales (ver Tabla I). Aquí están sobre representadas las empresas que no han introducido innovaciones de ningún tipo: cerca del $94 \%$ no lo ha hecho en materia comercial, el $87,5 \%$ en aspectos organizacionales, en tanto que la mitad no ha introducido innovaciones de proceso y más del $37 \%$ no lo ha hecho en productos. Teniendo en cuenta los resultados y la importancia de las innovaciones, están sobre representadas las empresas con un indicador global bajo de innovación, para casi el $70 \%$ de las empresas del grupo. Todo esto configura al grupo 5, como el menos innovador del análisis de la muestra. 


\begin{tabular}{|c|c|c|c|c|}
\hline Variable & $\begin{array}{l}\text { Modalida } \\
\text { d }\end{array}$ & $\begin{array}{c}\% \text { de la modalidad } \\
\text { en el grupo }\end{array}$ & $\begin{array}{l}\% \text { de la modalidad } \\
\text { en la muestra }\end{array}$ & $\underset{1}{\text { Sign. }}$ \\
\hline Aprendizaje interativo & Bajo & 100,00 & 17,98 & * \\
\hline Estructura CC & Baja & 68,75 & 15,73 & * \\
\hline Intensidad CC & Baja & 62,50 & $|4,6|$ & $*$ \\
\hline Compra externa de tecnología & Baja & 43,75 & 20,22 & $* *$ \\
\hline Vinculaciones & Baja & 62,50 & 35,96 & $* *$ \\
\hline Vinculaciones Nacionales & Baja & 43,75 & 21,35 & $* *$ \\
\hline Vinc nacionales por frecuencia & Baja & 37,50 & 15,73 & $* *$ \\
\hline Vinc nacionales por objetivo & Baja & 50,00 & 26,97 & $* *$ \\
\hline Vinc nacionales por agents & Baja & 37,50 & 17,98 & $* *$ \\
\hline Indicador de Innovación & Bajo & 68,75 & 37,08 & $*$ \\
\hline Innovación de Tipo Comercial & No & 93,75 & 64,04 & $*$ \\
\hline Innovación de Tipo Organizacional & No & 87,50 & 48,31 & * \\
\hline Innovación de Tipo Productos & No & 37,50 & 21,35 & $* * *$ \\
\hline Innovación de Tipo Procesos & No & 50,00 & 19,10 & $*$ \\
\hline \multicolumn{5}{|c|}{ Variables Subrepresentadas } \\
\hline Compra externa de tecnología & Alta & 12,50 & 33,71 & $* *$ \\
\hline Vinculaciones & Alta & 18,75 & 38,20 & $* * *$ \\
\hline Vinculaciones nacionales & Alta & 25,00 & 43,82 & $* * *$ \\
\hline Indicador de Innovación & Alto & 6,25 & 22,47 & $* * *$ \\
\hline
\end{tabular}

$\left({ }^{\prime}\right) *$ Significativa al I\%; ** Significativa al 5\%; *** Significativa al $10 \%$

Tabla 6. Principales Indicadores Sobre y Sub representados: Grupo 5.

Cerrando, en el Tabla 7 podemos ver resumidos los principales resultados del AFCM y de la clusterización lograda. Por un lado, podemos observar, que las firmas más innovativas (grupo I) son aquellas que recurren a altas fuentes domesticas de conocimiento y combinan este conocimiento con fuentes externas, como la compra externa de tecnología y vinculaciones internacionales. Esto muestra que el buen desempeño innovador de las empresas autopartistas depende de una determinada complementación entre fuentes domésticas de conocimiento (aprendizaje interno y vinculaciones nacionales) y externas o foráneas (compra externa de tecnología y vinculaciones internacionales), a pesar de desenvolverse en una trama productiva internacionalizada. - Esto último también puede apreciarse en los grupos intermedios (grupos 2, 3 y 4), donde la performance innovativa tiende a alcanzar niveles medios o promedio, $y$ está asociada a diversos balanceos entre fuentes externas e internas de conocimiento.

Por otro lado, vemos que en las firmas menos innovativas (grupo 5), tienen un bajo nivel de aprendizaje interactivo interno, de compra externa de tecnología y de vinculaciones nacionales, aunque cuentan con un nivel promedio de interacciones con agentes extranjeros. Esto sugiere que las empresas que no complementan su conocimiento externo con componentes domésticos y sus fuentes de conocimiento son principalmente externas tienden a tener una performance innovativa relativamente inferior $y$ que, incluso en tramas productivas profundamente internacionalizadas, las fuentes extranjeras de conocimiento no aparecen como un sustituto perfecto a las fuentes nacionales y de los procesos internos de aprendizaje. 


\begin{tabular}{|c|c|c|c|c|c|}
\hline & GRUPO I & GRUPO 2 & GRUPO 3 & GRUPO 4 & $\begin{array}{c}\text { GRUPO } \\
5\end{array}$ \\
\hline INNOVACIÓN & ALTA & - & - & MEDIA/BAJA & BAJA \\
\hline $\begin{array}{l}\text { APRENDIZAJE } \\
\text { INTERACTIVO }\end{array}$ & ALTO & ALTO & MEDIO & MEDIO & BAJO \\
\hline $\begin{array}{c}\text { Compra Externa de } \\
\text { Tecnología }\end{array}$ & MEDIA & ALTA & - & - & BAJA \\
\hline VINCULACIONES & ALTAS & BAJAS & MEDIAS & MEDIAS & BAJAS \\
\hline $\begin{array}{l}\text { VINCULACIONES } \\
\text { NACIONALES }\end{array}$ & ALTAS & BAJAS & MEDIAS & - & BAJAS \\
\hline $\begin{array}{l}\text { VINCULACIONES } \\
\text { INTERNACIONALES }\end{array}$ & MEDIAS & BAJAS & - & - & - \\
\hline
\end{tabular}

Tabla 7. Resumen de los Grupos.

\section{Conclusiones y Trabajo Futuro}

A partir de los resultados obtenidos, la principal conclusión de este trabajo es que la perfomance innovativa de las empresas autopartistas argentinas está positivamente relacionada con una complementación entre las fuentes internas y externas de obtención de conocimiento. Por otro lado, encontramos que, pese a que la internacionalización de la producción suele estar asociada con un mayor acceso a fuentes extranjeras de conocimiento, esto no minimiza ni extingue la importancia de las fuentes nacionales de obtención de conocimiento $y$, en ese sentido, la importancia del SNI es crucial para empresas establecidas dentro de la economía nacional, incluso para aquellas que operan en tramas productivas internacionalizadas y en un país en desarrollo, como lo es la Argentina.

Estos resultados deben ser corroborados en profundidad por investigación cualitativa (que es uno de los pasos siguientes en la investigación) y ser precisados incluyendo la consideración explícita de algunos aspectos del contexto 
institucional que han sido considerados de modo implícito en el análisis (como la organización de los mercados laborales y financieros, del sistema educativo, etc.), tarea que excede las posibilidades de este artículo. Además, teniendo en cuenta que la investigación incluye la incorporación del estudio comparativo de otra trama productiva, pero con distinto tipo de internacionalización, cobrarán relevancia otros aspectos institucionales (tales como la política regulatoria o sectorial) que no han sido tenido en cuenta en este análisis, pero son de suma importancia y que deberán ser incorporados.

\section{Consideraciones}

CEA CONICET (UNC) y FCE (UNC). Deben agradecerse muy especialmente todos los comentarios y sugerencias del Dr. Jorge Motta en la elaboración de este artículo y en la supervisión general de la investigación. Un avance de este estudio fue presentado como parte de la Ph.D. School on National Systems of Innovation and Economic Development de la Globelics Academy 2009 y, en ese sentido, deben agradecerse también todos los comentarios recibidos de los participantes de la academia, especialmente los de la Dra. Shulin Gu y el Dr. Sunil Mani. Todo error u omisión es responsabilidad exclusiva del autor, quien puede ser contactado a la siguiente dirección de e-mail: hernanmorero@eco.uncor.edu

\section{Referencias Bibliográficas}

ALBORNOZ, F., Milesi, D., Yoguel, G. (2005). Knowledge circulation in Vertically Integrated Production Networks: the cases of the Argentine Automotive and Iron and Steel industries. Innovation: Management, Policy and Practice, 3(23), 200-22I.

ANCORI, B., Bureth, A., Cohendet, P. (2000). The economics of knowledge: the debate about codification and tacit knowledge. Industrial and Corporate Change. 9(2).

ASHEIN, B., Gertler, M. (2004). The geography of innovation: regional innovation systems. En: Fagerberg, J., Mowery, D., Nelson, R. (eds.). The Oxford Handbook of Innovation. Oxford. Pp. 291-317.

APPENDINI, K., Nuijten, M. (2002). El papel de las instituciones en contextos locales. Revista de la CEPAL $N^{\circ} 76$.
BABBAGE, C. (I832). On the Economy of Machinery and Manufacturers. Charles Knight, London.

BARTHOLOMEW, S. (1997). National Systems of Biotechnology Innovation: Complex Interdependence in the Global System. Journal of International Business Studies 2(2), 24I-266.

BOSCHERINI, F., Yoguel, G. (2000). Aprendizaje y competencias como factores competitivos en el nuevo escenario. Algunas reflexiones desde la perspectiva de la empresa. En: Boscherini, F., Poma, L. (eds.). Territorio, conocimiento y competitividad de las Empresas. El rol de las instituciones en el espacio global. Miño y Dávila editores, Madrid.

BRESCHI, S., Malerba, F. (1997). Sectoral Innovation Systems: Technological Regimesn, Schumpeterian Dynamicas, and Spatial Boundaries. En: Edquist, C. (ed.). Systems of Innovation - Technologies, Institutions and Organizations. Pinter, London.

CANTWELL, J. (1997). The Globalisation of Technology: What Remains of the Product Cycle Model? En Archibugi, D., Michie, J. (eds.). Technology, Globalisation and Economic Performance. Cambridge University Press, New York. Pp. $215-240$.

CANTWELL, J., Piscitello L. (2000). Accumulating Technological Competence: Its Changing Impact on Corporate Diversification and Internationalization. Industrial and Corporate Change, 9(I), 2I-5I.

CARACOSTAS, P., Soete, L. (1997). The building of CrossBorder Institutions in Europe: Towards a European System of Innovation? En: Edquist, C. (ed.). Systems of Innovation Technologies, Institutions and Organizations. Pinter, London.

CARLSSON, B. (2006). Internationalization of Innovation Systems: A Survey of the Literature. Research Policy, 35(I), 56-67.

CARLSSON, B., Mudambi, R. (2003). Globalization, Entrepreneurship, and Public Policy: A Systems View. Industry and Innovation, 10(I), 103-1 16.

CHESNAI, F. (1986). Science, Technology and competitiviness. STI Review. OCDE, París. 
CHESNAI F. (198I). The notion of international competitiviness. Mimeo. OCDE, París.

COWAN, R., David, P., Foray, D. (2000). The explicit Economics of Knowledge Codification and Taciteness. Industrial and Corporate Change, 9 (2).

DOSI, G., SOETE, L. (1988). Technical Change and International Trade. En: Dosi, G., Freeman, C., Nelson, R., Silverberg, G., Soete, L. (eds.). Technical Change and Economic Theory. Francis Pinter y Columbia University Press, London y New York.

EDQUIST, C. (ed.) (1997). Systems of Innovation: Technologies, Institutions and Organization. Pinter, Londres.

EDQUIST, C. (200I). The Systems of Innovation Approach and Innovation Policy: an account of the state of the art. DRUID Conference, Aalborg.

ERNST, D., Lundvall, B. (1997). Information Technology in the Learning Economy. Challenges for Developing Countries. DRUID Woking Paper № 97/12. http://www3.druid.dk/wp//9970012.pdf [Acceso en marzo de 20I0].

FORAY, D. (1995). The Economics of Intellectual Property Rights and Systems of Innovation: The Persistence of National Practices Versus the New Global Model of Innovation. En: Hagedoorn, J. (ed.). Technical Change and the World Economy: Convergence and Divergence in Technology Strategies., E. Elgar, England. Pp. 109-I33.

FRANSMAN, M. (1999). Is National Technology Policy Obsolete in a Globalized World?. En Fransman, M. (ed.). The Japanese Vision. Visions of Innovation: The Firm and Japan. Oxford University Press, New York. pp. 167-20I.

FREEMAN, C. (1995). The National System of Innovation in Historical-Perspective. CJE, 19(I), 5-24.

FREEMAN, C. (1987). Technology and economic performance: lessons from Japan. Printer, Londres.

GRANSTRAND, O. (2000). Corporate Innovation Systems: A Comparative Study of Multi- Technology Corporations in Japan, Sweden and the USA. Chalmers University, Gothenburg.
LE BAS, C., Sierra, C. (2002). 'Location Versus Home Country Advantages' in R\&D Activities: Some Further Results on Multinationals' Locational Strategies. Research Policy, 3I (4), 589-609.

JENSEN, M. B., Johnson, B., Lorenz. E., Lundvall, B.-Å. (2007). Forms of knowledge and modes of innovation. Research Policy, 36(5), 680-693.

JOHNSON, B. (1992). Institutional Learning. En: Lundvall, B. (ed.) (1992). National Systems of Innovation: towards a theory of innovation and interactive learning. Pr. Ed.; Londres.

LIST, F. (I84I). Sistema Nacional de Economía Política. Fondo de Cultura Económica, México.

LÓPEZ, A. (2007). Desarrollo económico y Sistema Nacional de Innovación: el caso argentino de 1860 hasta 200I. Tesis Doctoral; UBA.

LUNDVALL, B. (1988). Innovation as an interactive process: from user-producer interaction $\circ$ the national system of innovation. En: Dosi, G. et al. (eds.). Technical Change and Economic Theory. Pinter, Londres y NY.

LUNDVALL, B. (ed.) (1992). National Systems of Innovation: towards a theory of innovation and interactive learning. Pr. Ed.; Lon.

LUNDVALL, B. (2007). National Innovation Systems: Analytical Concept and Development Tool. Industry \& Innovation, I4(I).

LUNDVALL, B., Johnson, B. (1994). The learning economy. Journal of Industry Studies, I (2).

LUNDVALL, B., Vang, J., Joseph, K., Chaminade, C. (2009). Bridging Innovation System Research and Development Studies: challenges and research opportunities. $7^{\text {th }}$ Globelics Conference, Senegal.

MALERBA, F. (2002). Sectoral systems of innovation and production. Research Policy, 3 I (2), 247-264.

MALERBA, F. (2004). Sectoral systems: how and why innovation differes across sectors. En: Fagerberg, J., Mowery, D., Nelson, R. (eds.). The Oxford Handbook of Innovation. OUP Oxford, Oxford. 
MOWERY, D. C., Oxley, J. (1997). Inward Technology Transfer and Competitiveness: The Role of National Innovation Systems. En: Archibugi, D., Michie, J. (eds.). Technology, Globalisation and Economic Performance.Cambridge University Press, New York and Melbourne. pp. I38-17I.

MOTTA, J., Barbero, M. (2007). Trayectoria de la industria automotriz en la Argentina desde sus inicios hasta fines de la década de 1990. En: Delfini, M., Dubbini, D., Lugones, M. y Rivero, I. (eds.). Innovación y empleo en tramas productivas de Argentina. UNGS. Prometeo Libros, Argentina.

MYTELKA, L. K. (2000). Local Systems of Innovation in a Globalized World Economy. Industry and Innovation, 7(I), 15-32.

NELSON, R. (1987). Understanding Technical Change as an Evolutionary Process. Elsevier, Amsterdam.

NELSON, R. (ed.) (1993). National Innovation Systems: A comparative analysis. Oxford University Press, NY.

NELSON, R., Rosenberg, N., (1993). Technical innovation and national systems. En: Nelson, R. (ed.) (1993). National Innovation Systems: A comparative analysis. Oxford University Press, NY.

NELSON, R., Winter, S. (1977). In search of a useful theory of innovation. Researh Policy, 6(I), 36-76.

NELSON R., Winter S. (1982). An Evolutionary Theory of Economic Change. The Belknap Press off Harvard University Press, Cambridge.

NIOSI, J., Bellon, B. (1994). The Global Interdependence of National Innovation Systems - Evidence, Limits, and Implications. Technology in Society, 16(2), 173-197.

NIOSI, J., Bellon, B. (1996). The Globalization of National Innovation Systems). En: De la Mothe y Paquet (eds.). Evolutionary Economics and the New International Political Economy. Pinter, NY. pp. I38-159.

NOVICK, M., Carrillo, J. (2006). Eslabonamientos Productivos Globales y Actores Locales: Debates y Experiencias en América Latina. En: De la Garza Toledo, E. (ed.), Teorías Sociales y Estudios del Trabajo; Antrhopos Editorial; UNAM, México.
OECD (1996). The Knowledge-Based Economy; OECD/CD (96) 102.

OHMAE, K. (1993). The end of the nation state: how region states harness the prosperity of the global economy. Free Press McMillan, New York.

PATEL, P., Pavitt, K, (199I). Large Firms in the Production of the Worlds Technology - an Important Case of NonGlobalization. Journal of International Business Studies, 22(I), I-2I.

PATEL, P. (1997). Localized Production of Technology for Global Markets. En: Archibugi y. Michie (eds.). Technology, Globalisation and Economic Performance. Cambridge University Press, New York and Melbourne. pp. 198-214.

PAVITT, K., Patel, P. (1999). Global Corporations and National Systems of Innovation: Who Dominates Whom?. En: Archibugi, Howells, J. y Michie (eds.). Innovation Policy in a Global Economy. Cambridge University Press, Cambridge. PP. 94-II9.

PAVITT, K. (1998). The Social Shaping of the National Science Base. Research Policy, 27(8), 793-805.

PEREZ, C. (1985). Microelectronics Long Waves and World Structural Change: New Perspectives for Developing Countries. World Development, I3(3).

SMITH, K. (2000). What is the 'the knowledge economy'? Knowledge intensive industries and distributed knowledge bases. DRUID Summer Conference.

SUNG, T.K., Carlsson, B. (2003). The Evolution of a Technological System: the Case of CNC Machine Tools in Korea. Journal of Evolutionary Economics, 13(4), 435-460.

YOGUEL, G. (ed.) (2002). Supplier development, productions environment and the creation of competitive advantages. The case of an iron and steel industrial network in Argentine. WP 1/2002. LITTEC. http://www.littec.ungs.edu.ar/pdfingles/Production\%20netw ork.\%20Siderca 19.pdf [Acceso en marzo de 2010] 


\section{Acerca del Autor}

Hernán Morero es Licenciado en Economía de la Universidad Nacional de Córdoba (UNC), Argentina. Actualmente realiza sus estudios de doctorado en economía en la UNC como becario del Consejo Nacional de Investigaciones Técnicas y Científicas (CONICET) en el Centro de Estudios Avanzados, llevando adelante el proyecto: "Procesos de aprendizaje en tramas productivas internacionalizadas y Sistema Nacional de Innovación". Su trabajo de investigación fue seleccionado para ser parte de la Globelics Academy 2009, la " 6 th Ph.D. School on National Systems of Innovation and Economic Development", que tuvo lugar en el ISEG de la UTL, Lisboa, Portugal. También es Profesor Ayudante en la Facultad de Ciencias Económicas de la UNC en Organización Industrial y Adscripto en Teoría Económica Evolucionista e Historia del Pensamiento Económico. Ha presentado diversos artículos vinculados a Economía de la Innovación en las reuniones de la Red PyMEs-MERCOSUR y a Historia del Pensamiento Económico en las reuniones de la Asociación Argentina de Economía Política. 